\title{
High harmonic generation and periodic level crossings
}

\author{
F. I. Gauthey, ${ }^{1,2}$ B. M. Garraway, ${ }^{1}$ and P. L. Knight ${ }^{1}$ \\ ${ }^{1}$ Optics Section, Blackett Laboratory, Imperial College, Prince Consort Road, London SW7 2BZ, United Kingdom \\ ${ }^{2}$ Laboratoire de Chimie-Physique, Matière et Rayonnement, Université Pierre et Marie Curie, Paris, France
}

(Received 3 February 1997)

\begin{abstract}
We propose a time-dependent level-crossing approach to the generation of high harmonics by a two-level system which stresses the periodicity of the processes involved. We establish relationships between multiple Landau-Zener crossings of energy levels and features of high harmonic generation such as cutoff, conditions for a plateau, and plateau height. [S1050-2947(97)04810-5]
\end{abstract}

PACS number(s): 42.50.Hz, 42.65.Ky, 32.80.Wr

The generation of high harmonics in the spectra of strongly laser-driven atoms has inspired much experimental and theoretical development in the past few years [1]. The generation of a broad plateau of harmonics appears to be most effective if the laser intensity is such that tunneling of electronic wave packets from the laser-deformed atomic potential is repeated many times in a phase-locked fashion. This observation provides the foundation for the very successful "recollision picture" [2-6]. Nevertheless, there are simple models of high harmonic generation which also predict a plateau and a cutoff, but have no apparent connection with the recollision model. One of these simple models is that of a two-state atomic system driven by a strong laser field whose frequency is much less than the atomic transition frequency, where the generic plateau and cutoff is once again reproduced [7-11]. However, this simple model contains no continuum and allows no possibility for any recollision processes, let alone many phase locked recollisions. The purpose of this paper is to introduce a time-dependent two-state model with periodic crossings of levels for the study of high harmonic generation. The model explicitly contains repeated encounters, phase locked to the laser driving field, which are remarkably reminiscent of the recollision model. We begin with a theoretical description of the driven two-level atom; then we establish the connection with periodic level crossings and present some numerical results in support of the new interpretation. Finally, we conclude with a comment on the connection with real atomic systems.

The theoretical description of the system begins with the time-dependent Hamiltonian for two coupled states with energy separation $\hbar \omega_{0}$ coupled to an electric field $\mathbf{E}(t)=\mathbf{E}_{0} \sin \left(\omega_{L} t\right)$,

$$
H(t)=\hbar\left[\begin{array}{lc}
-\omega_{0} / 2 & \Omega_{0} \sin \left(\omega_{L} t\right) \\
\Omega_{0} \sin \left(\omega_{L} t\right) & \omega_{0} / 2
\end{array}\right],
$$

where $\Omega_{0}$ denotes the Rabi frequency (or coupling constant), i.e., $\Omega_{0}=-\vec{\mu} \cdot \mathbf{E}_{0} / \hbar$ where $\vec{\mu}$ is the electric-dipole transition matrix element between the two states of the system. The laser frequency is $\omega_{L}$, and of course we do not make the rotating wave approximation. In this paper we ignore pulseshape effects, and will not assume that $\Omega_{0}$ is time dependent; thus the system is driven by a laser field with a constant amplitude. The state of the system can be represented by two amplitudes $c_{1}$ and $c_{2}$ so that $|\Psi(t)\rangle=c_{1}(t)|1\rangle+c_{2}(t)|2\rangle$ and, hence, in the basis of Eq. (1),

$$
|\Psi(t)\rangle=\left(\begin{array}{l}
c_{1}(t) \\
c_{2}(t)
\end{array}\right)
$$

We will be interested in determining the generic origin of the plateau and cutoff features observed in the high harmonic generation (HHG) spectra. As these features are characteristic features in all HHG spectra, and appear in both the coherent and incoherent parts of each HHG spectrum, we restrict our study to the coherent part of the spectrum $S_{C}(\omega)$, i.e., the spectrum of the mean dipole, which is predominant for forward detection [12]. Thus the main observable of interest is the time-dependent mean dipole moment,

$$
d(t)=\mu\left[c_{1}^{*}(t) c_{2}(t)+c_{1}(t) c_{2}^{*}(t)\right]
$$

which leads to the coherent part of the spectrum [12],

$$
S_{C}(\omega)=\left|\int d t e^{-i \omega t} d(t)\right|^{2} .
$$

The numerical solution of the problem is straightforward: the Schrödinger equation and the Hamiltonian (1) result in coupled differential equations which are readily solved.

In order to present a picture of "level crossings" we perform a very simple basis rotation by utilizing the unitary operator

$$
\hat{U}=\frac{1}{\sqrt{2}}\left[\begin{array}{rr}
1 & 1 \\
-1 & 1
\end{array}\right] .
$$

Then the Hamiltonian $H(t)$ is expressed in the rotated basis as

$$
\widetilde{H}(t)=\hat{U} H(t) \hat{U}^{\dagger}=\hbar\left[\begin{array}{cc}
\Omega_{0} \sin \left(\omega_{L} t\right) & \omega_{0} / 2 \\
\omega_{0} / 2 & -\Omega_{0} \sin \left(\omega_{L} t\right)
\end{array}\right],
$$

and we see that the rotation exchanges the roles of Rabi frequency and atomic frequency. In this basis, the diagonal terms display the time dependence $\sin \left(\omega_{L} t\right)$, and the offdiagonal terms $\omega_{0} / 2$ correspond to the coupling between those two time-dependent states. We see that we thus obtain a picture of periodic crossings [13-15] between the trans- 


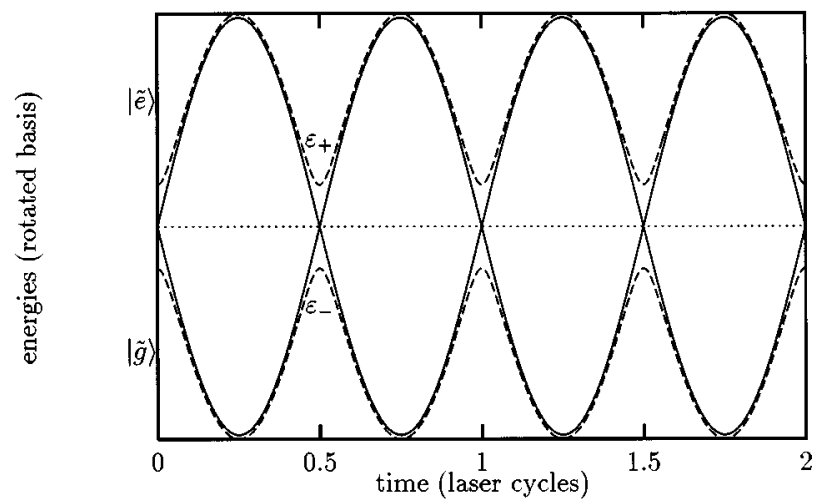

FIG. 1. The energies of the transformed states $|\widetilde{e}\rangle$ and $|\widetilde{g}\rangle$ of the rotated basis are represented as a function of time. The time unit is the laser cycle. We clearly note periodic crossings at each halfcycle of the laser $T_{1 / 2}=\pi / \omega_{L}$. The dotted lines correspond to the instantaneous quasienergies $\varepsilon_{+}$and $\varepsilon_{-}$, which are the eigenvalues of the transformed Hamiltonian (6).

formed states of the two-level system; see Fig. 1. Each crossing occurs at a time for which $\Omega_{0} \sin \left(\omega_{L} t\right)=0$ and the time between each crossing is determined by half the period of the laser frequency, $T_{1 / 2}=\pi / \omega_{L}$, just as in the recollision model. We will see later that a time $t_{c}$ characterizes the duration of a crossing. This way of formulating the problem will allow us to relate it to the nonadiabatic techniques utilized in Ref. [13] and [16]. The transformation (5) matches that in Refs. [17] where studies were made of two-electron dissociative ionization and above-threshold dissociation using classical trajectories with surface hopping. However, in this paper we consider the consequences for high harmonic generation of a simple two-level system where coherence is propagated between the level crossings. Further, the crossings we describe here should not be confused with the crossings of Floquet states found when describing pulse-shape effects [18]. Finally, we note that in the rotated basis the dipole moment belonging to the system (1) becomes the population difference. That is,

$$
\begin{aligned}
d(t) & =\mu\left\langle\widetilde{\Psi}(t)\left|\hat{U}(|2\rangle\langle 1|+| 1\rangle\langle 2|) \hat{U}^{\dagger}\right| \widetilde{\Psi}(t)\right\rangle \\
& =\mu\left(\left|\widetilde{c_{1}}(t)\right|^{2}-\left|\widetilde{c_{2}}(t)\right|^{2}\right),
\end{aligned}
$$

where

$$
|\widetilde{\Psi}(t)\rangle=\hat{U}|\Psi(t)\rangle=\left(\begin{array}{c}
\widetilde{c_{1}}(t) \\
\widetilde{c_{2}}(t)
\end{array}\right)=\frac{1}{\sqrt{2}}\left(\begin{array}{c}
c_{1}+c_{2} \\
-c_{1}+c_{2}
\end{array}\right) .
$$

Figure 2 shows an example of the behavior of the mean dipole as a function of the time (in units of the laser cycle), i.e., the population inversion in the rotated basis [see Eq. (7)]. We clearly see that the populations are inverted at each half laser cycle, that is at each level crossing in the rotated basis. This is the signature of adiabatic passage through each crossing, and is a periodic effect. We also note that, between each crossing, there are oscillations which have amplitudes that vary between crossings, and from crossing to crossing. These arise because, although we may have nonadiabatic ef-

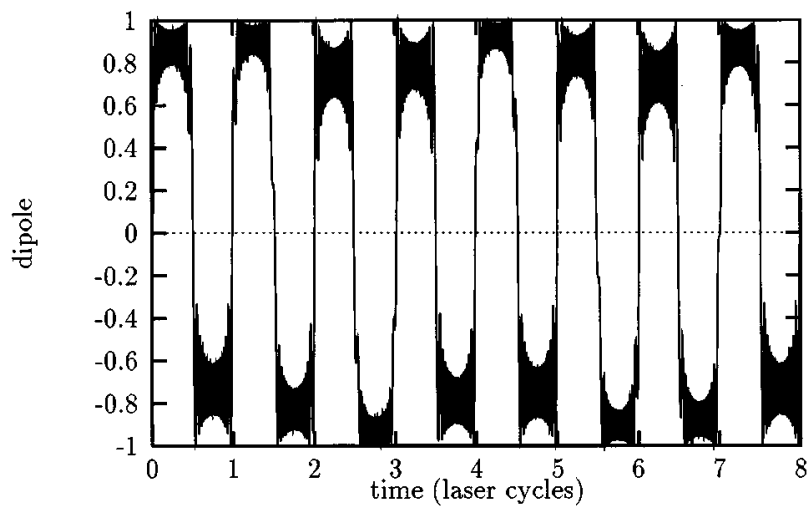

FIG. 2. The time-dependent dipole, or population inversion in the rotated basis, is shown as a function of time. The initial state of the atom is the ground state and there is a Rabi frequency $\Omega_{0} \simeq 0.8$ a.u., a laser frequency $\omega_{L}=0.0258$ a.u., and an atomic frequency $\omega_{0}=0.3$ a.u. These parameters are chosen so that the adiabaticity parameter $\Lambda=1.1$, and the crossing parameter $R=0.1892$ in the level-crossing picture. The time unit is the laser cycle.

fects at a crossing, away from the crossing we have adiabatic following where the instantaneous energy of the system is given by the diagonal form of Eqs. (1) and (6):

$$
\mathcal{E}_{ \pm}(t)= \pm \hbar \sqrt{\left(\omega_{0} / 2\right)^{2}+\left(\Omega_{0} \sin \omega_{L} t\right)^{2}} .
$$

The "quasienergies" $\mathcal{E}_{ \pm}(t)$ are indicated by the dashed lines in Fig. 1. In the Bloch vector picture of a two-level system the Bloch vector, representing the state of the system, precesses about a moving vector with coordinates given by diagonal and off-diagonal terms in the Hamiltonian. The population difference is conventionally given by the projection of the Bloch vector on the $z$ axis, and this results in the oscillations changing in amplitude (due to the changing projection angle) and in frequency (as the quasienergy increases). A straightforward argument $[19,14]$ shows that the population difference (dipole moment) between the crossings will be given by the adiabatic following solution:

$$
\begin{aligned}
d(t)= & \mu\left[\left(\left|a_{1}\right|^{2}-\left|a_{2}\right|^{2}\right) \cos 2 \theta(t)\right. \\
& \left.+2 \sin 2 \theta(t) \operatorname{Re}\left(a_{1} a_{2}^{*} e^{i \phi(t)}\right)\right],
\end{aligned}
$$

where $a_{1}$ and $a_{2}$ are the amplitudes of the adiabatic states (which change at each crossing), the angle $\theta(t)$ is defined by

$$
\cos 2 \theta(t)=\frac{\Omega_{0} \sin \omega_{L} t}{\sqrt{\left(\omega_{0} / 2\right)^{2}+\left(\Omega_{0} \sin \omega_{L} t\right)^{2}}},
$$

and the adiabatic phase $\phi(t)$ follows from the integration of the difference between the adiabatic energies:

$$
\phi(t)=\int_{0}^{t}\left[\mathcal{E}_{+}(t)-\mathcal{E}_{-}(t)\right] d t=\frac{2 \Omega_{0}}{k \omega_{L}} E\left(\omega_{L} t, k\right),
$$

where $E\left(\omega_{L} t, k\right)$ is an incomplete elliptic integral of the second kind, and

$$
k=\Omega_{0} / \sqrt{\left(\omega_{0} / 2\right)^{2}+\Omega_{0}^{2}} .
$$


The changes in the amplitudes of the oscillations between crossings (see Fig. 2) are directly related to nonadiabatic effects occurring at each crossing. The crossing mixes the adiabatic states, and thus changes the initial conditions for the adiabatic following.

Time-dependent level crossings have many physical realizations such as atomic collisions [20,21], two-level systems driven by frequency-modulated light, microwave-induced Stark effects [22], molecular dissociation [17], and in the study of molecular wave-packet dynamics [16,23]. Such models have also been useful in laser cooling and trapping $[24,13]$. A useful approach in all of these systems is to assume that the crossing time $t_{c}$ over which the crossing takes place is so short when compared to the time betwen the crossings, as we will see, that we may linearise the Hamiltonian (6) over a small time interval $\Delta t$ [25]

$$
\widetilde{H}(t)=\hbar\left(\begin{array}{lr}
\Omega_{0} \omega_{L} \Delta t & \omega_{0} / 2 \\
\omega_{0} / 2 & -\Omega_{0} \omega_{L} \Delta t
\end{array}\right) .
$$

The mathematical advantage of this kind of linearization is that we can determine how the wave function $\Psi$ is changed because of the "coupling" $\omega_{0} / 2$. The key parameter in describing this is the adiabaticity

$$
\Lambda=\frac{\left(\omega_{0} / 2\right)^{2}}{\Omega_{0} \omega_{L}},
$$

i.e., the ratio of the square of the coupling to the energy slope at the crossing in the rotated basis. The usefulness of this parameter is seen if we determine the probability $P$ of remaining in the same (rotated basis) state at a linear crossing which is the Landau-Zener transition probability [25]

$$
P=\exp (-\pi \Lambda)
$$

For adiabatic crossings $\Lambda \gg 1$ and $P \rightarrow 0$, while for "diabatic" (or "nonadiabatic") crossings, $\Lambda \ll 1$, and $P \rightarrow 1$. We note that from the point of view of level crossings, the adiabatic regime $(\Lambda \rightarrow \infty)$ corresponds to what is actually called the perturbative regime in the original basis of the Hamiltonian (1). This is because $\Omega_{0} \rightarrow 0$ corresponds to $\Lambda \rightarrow \infty$ in Eq. (15).

The condition ensuring that the Landau-Zener concept is applicable in our model is that the crossings should be well localized or well separated from each other. In the case where this condition is not satisfied, each crossing region, with its strong mixing of two levels, would extend to the next crossing, and this would invalidate the Landau-Zener approach to the crossing. We also find that, unless the crossings are well separated, there is no plateau for the harmonic spectrum. In order to quantify this we need an expression for the time over which a crossing is effective, $t_{c}$. A suitable definition of $t_{c}$ is given by the time at which the magnitude of the diagonal and off-diagonal elements of Eq. (6) become equal, i.e.,

$$
t_{c}=\frac{\omega_{0} / 2}{\Omega_{0} \omega_{L}} .
$$

Then the condition for well-separated crossings is [13]

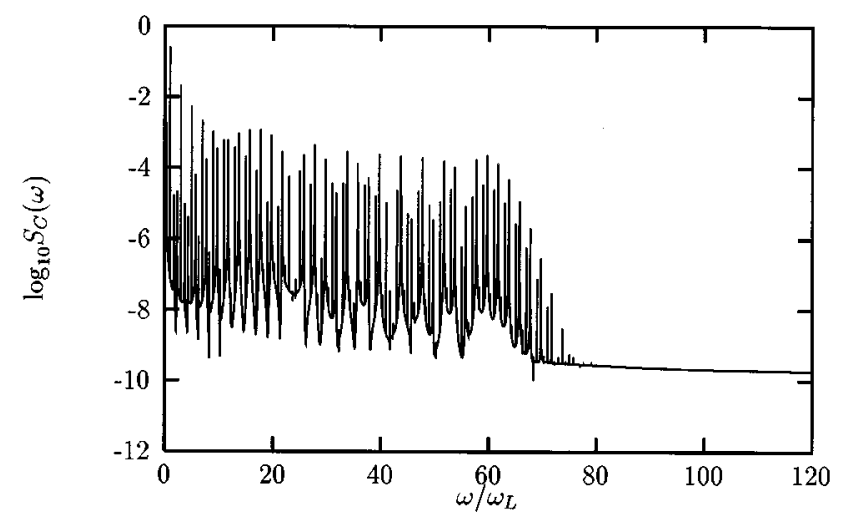

FIG. 3. Logarithm of the coherent spectrum $S_{C}(\omega)$ [Eq. (4)] is shown for the parameters in Fig. 2 and for 64 cycles of the laser field. The frequency unit is the laser frequency.

$$
t_{c} \ll \frac{\pi}{\omega_{L}},
$$

which can be written as a condition for a dimensionless crossing parameter $R$, which is

$$
R=\omega_{0} / 2 \Omega_{0},
$$

and must satisfy $R \ll \pi$. This corresponds to a weak-coupling regime in the rotated basis, as is seen in the expression of the transformed Hamiltonian $\widetilde{H}(t)$ [see Eq. (6)]. Note that this condition is satisfied when the system is excited with very intense laser fields, that is, for high Rabi frequencies $\Omega_{0}$ which corresponds to a strong coupling in the original (unrotated) basis, and is in the region of parameter space of interest.

We now focus on the harmonic spectrum for the case where the two-level system is initially in its ground state, and then submitted to a laser square pulse with a duration of 64 cycles and with a laser frequency $\omega_{L}=0.0258$ a.u. The first part of the time evolution of the dipole is shown in Fig. 2, and in Fig. 3 we present the coherent spectrum obtained for a Rabi frequency $\Omega_{0} \simeq 0.8$ a.u. The corresponding adiabaticity parameter has a value of $\Lambda=1.1$, and the crossing parameter is $R=0.1892$ which is short enough to ensure separated crossings. The spectrum presents odd harmonics of the laser frequency and hyper-Raman lines. We see that the harmonic spectrum shows a plateau and a cutoff which is defined, as usual, to lie at the end of the plateau $[2-5,7,9,10]$.

The value of the cutoff can be easily understood from the picture of adiabatic following. We see in Eq. (10) that the last term can introduce rapid oscillations into the dipole moment because of the adiabatic phase $\phi(t)$. The instantaneous frequency is given by the difference of the quasienergies, Eq. (9). That difference has a minimum value of $\omega_{0}$ and a maximum value of

$$
q_{\max } \omega_{L}=2 \sqrt{\left(\omega_{0} / 2\right)^{2}+\Omega_{0}^{2}}
$$

where $q_{\max }$ defines the maximum order of the harmonic. This expression agrees with an approximate result given in Ref. [10]. We can rewrite Eq. (20) in terms of the adiabaticity parameter as 


$$
q_{\max }=2 \frac{\Lambda}{R}\left(1+\frac{1}{R^{2}}\right)^{1 / 2},
$$

so that the position of cutoff is seen to increase linearly with adiabaticity $\Lambda$. For well-separated crossings $q_{\max }$ is inversely proportional to the square of the crossing time $t_{c}$. We find in our numerical work that the simple cutoff law Eq. (20) works very well for all cases that present a plateau in the spectrum. For the parameters of Fig. 2 the predicted cutoff is at $q_{\text {max }} \approx 63$, in agreement with the figure.

We may observe the effect of changing the laser frequency $\omega_{L}$ on the plateau and cutoff observed in HHG spectra, and relate these variations to the adiabaticity parameter $\Lambda$ (as the crossing parameter $R$ is independent of $\omega_{L}$ in the level-crossing model picture). As an example, here we consider a doubling of the laser frequency so that $\omega_{L}=0.0516$ a.u.; this corresponds to an adiabaticity parameter $\Lambda=0.55$ and the same crossing time-scale parameter $R$. The reduction in the adiabaticity parameter results in greater nonadiabatic effects which mean that we no longer have population swapping in time because the transfer of population between the adiabatic states is incomplete. The resulting behavior can be described by "gross structures" [14] which periodically shift the fast adiabatic evolution. The spectrum has a plateau which is very much reduced and has a cutoff at $q_{\max } \simeq 31$, in agreement with the cutoff law (20). In this case, and in general, we have found that the plateau height is proportional to $\exp (-\pi \Lambda)$-although there are quite large fluctuations about this value (a topic which may be an interesting subject for further study). Thus the characteristics of the plateau are strongly related to the Landau-Zener transition probability (16) and the value of the adiabaticity parameter $\Lambda$.

To summarize, we have seen that the origin of the plateau is linked to rapid crossings of the levels at each half-cycle of the laser period, during which nonadiabatic effects take place. We have also found that the cutoff for the plateau is accurately given from the picture of adiabatic following by expression (20), and the height of the plateau is approximately proportional to the Landau-Zener transition probability (16). The duration of the crossings $t_{c}$ is crucial in this description as it allows nonadiabatic effects to take place at regular intervals. This means that an essential condition for the appearance of a plateau is given by Eq. (18), which ensures that the duration of a crossing is much shorter than the time between crossings. This suggests an immediate analogy to the recollision picture $[2-4,6]$ where we also experience two distinct time scales: a short interaction time in the vicinity of the nucleus (during which the electron may recombine), and a longer time during which the electron is driven as a free electron in the continuum under the action of the laser field.

This work was supported by the United Kingdom Engineering and Physical Sciences Research Council, and by the European Union.
[1] A. L'Huillier, L. A. Lompré, G. Mainfray, and C. Manus, in Atoms in Intense Laser Fields, edited by M. Gavrila (Academic, Boston, 1992); M. Protopapas, C. H. Keitel, and P. L. Knight, Rep. Prog. Phys. 60, 389 (1997).

[2] J. L. Krause, K. J. Schafer, and K. C. Kulander, Phys. Rev. Lett. 68, 3535 (1992).

[3] P. B. Corkum, Phys. Rev. Lett. 71, 1994 (1993).

[4] K. C. Kulander, K. J. Schafer, and J. L. Krause, in Super Intense Laser-Atom Physics, Vol. 316 of NATO Advanced Study Institute, Series B: Physics, edited by B. Piraux, A. L'Huillier, and K. Rzążewski, (Plenum, New York, 1993), p. 95.

[5] M. Lewenstein, Ph. Balcou, M. Yu. Ivanov, A. L'Huillier, and P. B. Corkum, Phys. Rev. A 49, 2117 (1994).

[6] M. Protopapas, D. G. Lappas, C. H. Keitel, and P. L. Knight, Phys. Rev. A 53, R2933 (1996).

[7] B. Sundaram and P. W. Milonni, Phys. Rev. A 41, 6571 (1990).

[8] L. Plaja and L. Roso, J. Mod. Opt. 40, 793 (1993).

[9] M. Yu. Ivanov, and P. B. Corkum, Phys. Rev. A 48, 580 (1993).

[10] A. E. Kaplan and P. L. Shkolnikov, Phys. Rev. A 49, 1275 (1994).

[11] F. I. Gauthey, C. H. Keitel, P. L. Knight, and A. Maquet, Phys. Rev. A 55, 615 (1997) and references therein.

[12] F. I. Gauthey, C. H. Keitel, P. L. Knight, and A. Maquet, Phys. Rev. A 52, 525 (1995) and references therein.

[13] B. M. Garraway and S. Stenholm, Phys. Rev. A 45, 364 (1992).
[14] B. M. Garraway and N. V. Vitanov, Phys. Rev. A 55, 4418 (1997).

[15] G. S. Agarwal and W. Harshawardhan, Phys. Rev. A 50, R4465 (1994).

[16] B. M. Garraway and K.-A. Suominen, Rep. Prog. Phys. 58, 365 (1995).

[17] P. Dietrich, M. Yu. Ivanov, F. A. Ilkov, and P. B. Corkum, Phys. Rev. Lett. 77, 4150 (1996); M. Thachuk, M. Yu. Ivanov, and D. M. Wardlaw, J. Chem. Phys. 105, 4094 (1996).

[18] M. L. Pons, R. Taieb, and A. Maquet, Phys. Rev. A 54, 3634 (1996).

[19] L. Allen and J. Eberly Optical Resonance and Two-Level Atoms (Dover, New York, 1975).

[20] E. E. Nikitin and S. Ya. Umanskii, Theory of Slow Atomic Collisions (Springer, Berlin, 1984).

[21] D. S. F. Crothers, Adv. Phys. 20, 405 (1971); Adv. At. Mol. Phys. 17, 55 (1981); Phys. Scr. 40, 634 (1989).

[22] W. van de Water, S. Yoakum, T. van Leeuwen, B. E. Sauer, L. Moorman, E. J. Galvez, D. R. Mariani, and P. M. Koch, Phys. Rev. A 42, 572 (1990).

[23] B. M. Garraway and S. Stenholm, Opt. Commun. 83, 349 (1991); K.-A. Suominen, B. M. Garraway, and S. Stenholm, Phys. Rev. A 45, 3060 (1992).

[24] A. P. Kazantsev, G. I. Surdutovich, and V. P. Yakovlev, Mechanical Action of Light on Atoms (World Scientific, Singapore, 1990).

[25] L. D. Landau, Phys. Z. Sowjetunion 2, 46 (1932); C. Zener, Proc. R. Soc. London, Ser. A 137, 696 (1932). 